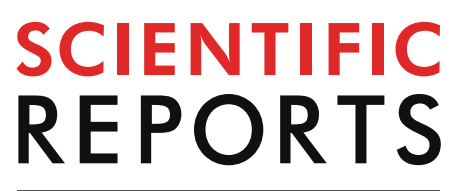

natureresearch

Check for updates

\title{
Impaired chondrocyte U3 snoRNA expression in osteoarthritis impacts the chondrocyte protein translation apparatus
}

Ellen G. J. Ripmeester ${ }^{1}$, Marjolein M. J. Caron ${ }^{1}$, G. G. H. van den Akker ${ }^{1}$, Don A. M. Surtel ${ }^{1}$, Andy Cremers ${ }^{1}$, Panagiotis Balaskas ${ }^{3}$, Philip Dyer ${ }^{3}$, Bas A. C. Housmans ${ }^{1}$, Alzbeta Chabronova ${ }^{1}$, Aibek Smagul ${ }^{3}$, Yongxiang Fang ${ }^{3}$, Lodewijk W. van Rhijn ${ }^{1,2}$, Mandy J. Peffers ${ }^{3,4}$ \& Tim J. M. Welting ${ }^{1,2,4 凶}$

\begin{abstract}
Although pathways controlling ribosome activity have been described to regulate chondrocyte homeostasis in osteoarthritis, ribosome biogenesis in osteoarthritis is unexplored. We hypothesized that U3 snoRNA, a non-coding RNA involved in ribosomal RNA maturation, is critical for chondrocyte protein translation capacity in osteoarthritis. U3 snoRNA was one of a number of snoRNAs with decreased expression in osteoarthritic cartilage and osteoarthritic chondrocytes. OA synovial fluid impacted U3 snoRNA expression by affecting U3 snoRNA gene promoter activity, while BMP7 was able to increase its expression. Altering U3 snoRNA expression resulted in changes in chondrocyte phenotype. Interference with U3 snoRNA expression led to reduction of rRNA levels and translational capacity, whilst induced expression of U3 snoRNA was accompanied by increased 185 and $28 \mathrm{~S}$ rRNA levels and elevated protein translation. Whole proteome analysis revealed a global impact of reduced U3 snoRNA expression on protein translational processes and inflammatory pathways. For the first time we demonstrate implications of a snoRNA in osteoarthritis chondrocyte biology and investigated its role in the chondrocyte differentiation status, rRNA levels and protein translational capacity.
\end{abstract}

Osteoarthritis $(\mathrm{OA})$ is a chronic debilitating joint disease that is strongly associated with ageing ${ }^{1,2}$. OA involves pathological cellular processes in all joint structures and affects articular cartilage integrity, leading to dysfunctional joint articulation ${ }^{2}$. During OA development and progression, the articular chondrocyte's phenotype changes $^{3-5}$ and presents with disturbed cellular homeostasis characterized by abnormal expression of (pre-) hypertrophic-[RUNX2 (runt-related transcription factor 2); COL10A1 (type X collagen)], catabolic-[ALPL (alkaline phosphatase); MMP13 (matrix metallopeptidase 13) and ADAMTS5 (a disintegrin and metalloproteinase with thrombospondin motifs 5) and inflammatory (COX2 (cyclooxygenase 2) and IL-6 (interleukin 6)] genes, while chondrogenic gene expression [SOX9 (SRY-box transcription factor 9); COL2A1 (type 2 collagen); ACAN (aggrecan) and NKX3-2 (NK3 homeobox 2)] is attenuated ${ }^{3,4}$. The biomolecular processes that catalyze disturbances in the articular chondrocyte phenotype leading to OA are poorly understood, and it is expected that a comprehensive understanding of the avenues leading to disruption of articular chondrocyte homeostasis will provide important clues for future treatments.

Chondrocytes are specialized secretory cells, enabling the synthesis and maintenance of the protein-rich cartilage extracellular matrix (ECM). Disturbances in chondrocyte protein translation in cartilage development and OA are connected to mTOR (mammalian target of rapamycin) activity ${ }^{6}$, endoplasmic reticulum stress ${ }^{7}$, unfolded protein response and apoptosis ${ }^{8}$. These responses change the downstream translational activity of the

\footnotetext{
${ }^{1}$ Laboratory for Experimental Orthopedics, Department of Orthopedic Surgery, Maastricht University, Universiteitssingel 50, 6229 ER Maastricht, The Netherlands. 'Laboratory for Experimental Orthopedics, Department of Orthopedic Surgery, Maastricht University Medical Center, P.O. Box 5800, 6202 AZ Maastricht, The Netherlands. ${ }^{3}$ Department of Musculoskeletal Biology, Institute of Ageing and Chronic Disease, University of Liverpool, Liverpool L69 3BX, UK. ${ }^{4}$ These authors contributed equally: Mandy J. Peffers and Tim J. M. Welting. ${ }^{\circledR}$ email: t.welting@maastrichtuniversity.nl
} 
biosynthesized ribosome. The assembled mammalian ribosome is built from ribosomal RNAs (rRNAs), together with at least 80 different protein subunits ${ }^{9}$. In the ribosome the $18 \mathrm{~S}$ rRNA guides the decoding of the mRNA message, while the 28S rRNA forms the core of the peptidyltransferase center that polymerizes the amino acid sequence encoded by the mRNA into functional proteins. Post-transcriptional maturation of rRNAs is an integral part of the biosynthesis of ribosomes ${ }^{10-13}$ and ribonucleolytic processing of the major 47S rRNA precursor into mature $18 \mathrm{~S}, 5.8 \mathrm{~S}$, and $28 \mathrm{~S}$ rRNAs is rate-limiting for ribosome biogenesis ${ }^{10}$. The U3 small nucleolar RNA (snoRNA) is a highly abundant and evolutionarily conserved box C/D-class snoRNA, guiding the endoribonucleolytic processing of the 5' external transcribed spacer (ETS) of the 47S pre-rRNA by base complementarityguided pre-rRNA substrate recognition ${ }^{14}$ and plays a key role in the maturation of $18 \mathrm{~S} \mathrm{rRNA}^{12,15}$. Although extensively studied in yeast ${ }^{16}$, it was only recently demonstrated that U3 snoRNA is indispensable for rRNA maturation in human cells ${ }^{16}$.

Pathways controlling ribosome activity have been described in the regulation of chondrocyte homeostasis ${ }^{17,18}$. Here, we postulated that not only ribosome activity is involved in chondrocyte homeostasis, but that OA pathophysiological situations can cause alterations in chondrocyte ribosome biogenesis with consequences for cellular protein translation. In this study we discovered that the chondrocyte expression of ribosome biogenesis factor U3 snoRNA is impacted in OA cartilage and we investigated its connection with OA pathophysiological conditions in chondrocytes and its role in influencing rRNA levels and chondrocyte translation capacity.

\section{Materials and methods}

Cell culture. Human articular chondrocytes (HACs) were isolated from femoral cartilage from total knee arthroplasty for end-stage OA (OA-HACs; average age 68.5 years with SD 8.2 years). Non-OA chondrocytes were isolated from cartilage of non-OA individuals undergoing anterior cruciate ligament repair (average age 25.7 years with SD 17.9 years). Medical ethical approval for collecting and using HACs was received from the Medical Ethics Committee from the Maastricht University Medical Center (approval number 2017-0183). Dutch medical ethical guidelines were followed for studying human samples and informed consent was acquired. Chondrocyte isolation and culture was performed as described previously ${ }^{5}$. SW1353 cells were purchased from ATCC (ATCC; Middlesex, UK) and cultured in a humidified atmosphere at $37^{\circ} \mathrm{C}, 5 \% \mathrm{CO}_{2}$. Culture medium was Dulbecco's Modified Eagle Medium (DMEM)/F-12 (Life Technologies, Waltham, Massachusetts, USA) supplemented with $10 \%$ fetal calf serum (FCS; Sigma-Aldrich, Dorset, UK), 1\% non-essential amino acids (NEAA; Life Technologies) and $1 \%$ penicillin/streptomycin (P/S, Invitrogen Life Technologies). In experiments non-OA chondrocytes were exposed to control $(20 \%(\mathrm{v} / \mathrm{v})$ of $0.9 \% \mathrm{NaCl})$, non-OA synovial fluid (non-OA SF; $20 \%(\mathrm{v} / \mathrm{v})$; pool of ten donors; average age 56.7 years with SD 7.5 years; purchased from Articular Engineering, Northbrook, Illinois, USA) or OA synovial fluid (OA-SF; $20 \%$ (v/v); pool of six or ten donors; for ten-donors-pool average age 65.4 years with SD 9.1 years; for six-donor-pool average age 73.0 years with SD 6.8 years; MEC approval 20170183). The average age difference between the ten-donor-pools was not statistically significant (Mann Whitney, $\mathrm{p}=0.069$ ). SW1353 and non-OA HACs were exposed to BMP7 (R\&D Systems, Minneapolis, Minnesota, USA).

Microarray. Cartilage biopsies were obtained from the femoral intercondylar notch of non-OA male human knees of young individuals undergoing anterior cruciate ligament repair surgery $(n=6$; mean age $\pm S D$ $22.7 \pm 4.1$ years). OA cartilage was obtained from male OA patients undergoing total knee arthroplasty $(\mathrm{n}=6$; mean age $66.4 \pm 15.9$ years). Sample characteristics; sample collection; RNA isolation of OA and healthy cartilage; the micro-array and data analysis have been performed as previously reported ${ }^{19}$. The heat map of hierarchical clusters of correlations and PCA plot are shown in Supplementary Fig. 1. Medical ethical approval for collecting and using cartilage biopsies was received from the Medical Ethics Committee from the Maastricht University Medical Center (approval number 08-04-028 and 14-4-038). Dutch medical ethical guidelines were followed for studying human samples and informed consent was acquired. Sequence data have been submitted to National Centre for Biotechnology Information Gene Expression Omnibus (NCBI GEO); E-MTAB-5715.

DMM. Mice (C57BL/6) were group housed in individually ventilated cages at a $12 \mathrm{~h}$ light/dark cycle, with ad libitum access to food and water. Under anesthesia, a $3 \mathrm{~mm}$ skin incision was made over the medial aspect of the patellar ligament through the joint capsule into the femorotibial joint of the left knee. The medial meniscotibial ligament was transected to destabilize the cranial pole of the medial meniscus from the anterior tibial plateau. In sham-operated mice $(n=3)$ the medial meniscotibial ligament was visualized but not transected. Mice were sacrificed 8 weeks post-surgery and stifles were fixated in phosphate-buffered $3.7 \%$ formalin. Ethical review was conducted by the University of Liverpool Animal Welfare and Ethical Review Body and ethical approval was obtained from the University of Liverpool (project license P74DC0667). All experimental protocols were performed in compliance with the UK Animals (Scientific Procedures) Act 1986 regulations.

U3 snoRNA in situ hybridization. Dissected stifles were decalcified in a 1:1 10\% formalin (VWR, Radnor, Pennsylvania, USA): $200 \mathrm{mM}$ EDTA (VWR) for 3 weeks, embedded in paraffin and sectioned at $5 \mu \mathrm{m}$, three adjacent sections per slide. Following dewaxing sections were circled with a hydrophobic pen (Agilent, Santa Clara, California, US). U3 snoRNA in situ hybridization was performed following the manufacturer's instructions of the miRCURY LNA miRNA ISH Optimization kit (Qiagen, Hilden, Germany). Probe sequences (Eurogentec, Liège, Belgium) are shown in Supplementary Table 1. Detection was performed by $\sim 1 \mathrm{~h}$ incubation at $37^{\circ} \mathrm{C}$ with NBT/BCIP (Roche, Basel, Switzerland). Slides were washed with PBS-Tween (PBS-T; phosphate buffered saline with $0.1 \%$ Tween-20), counterstained 5 min 0.05\% FastGreen, 5 min 0.05\% Safranin O, dehydrated to xylene as standard and mounted with Pertex Mounting Medium. 
Knockdown and ectopic expression of U3 snoRNA. Transfection of non-OA HACs or SW1353 cells (seeded at 30,000 or 20,000 cells $/ \mathrm{cm}^{2}$, respectively) with $100 \mathrm{nM}$ of U3 snoRNA antisense oligonucleotide (ASO) or a scrambled version thereof (SCR) was performed using HiPerfect (Qiagen) according to the manufacturers' protocol. Non-OA HACs were transfected after $8 \mathrm{~h}$ of prior serum starvation, while SW1353 cells were transfected without prior serum starvation. Sequences of U3 snoRNA ASO and SCR (Eurogentec, Liège, Belgium) are shown in Supplementary Table 2. Transfection of non-OA HACs with a U3 snoRNA mini-gene was performed using Fugene6, following the manufacturers' protocol (Promega, Madison, Wisconsin, USA). The U3 snoRNA mini-gene was synthesized (Genecust, Boynes, France) and cloned into the pUC57 vector. The mini-gene consisted of the endogenous 500 nucleotide sequence upstream of the U3 snoRNA transcription start site, the preU3 snoRNA sequence and followed by 250 nucleotides downstream of the transcription termination sequence. The U3 mini-gene construct was transfected at $10 \mathrm{ng}$ plasmid $/ \mathrm{cm}^{2}$ and because of the low amount of U3 snoRNA mini-gene supplemented with pGluc-basic-2-CMV (NEB, Ipswich, Massachusetts, USA) as carrier plasmid. In control conditions, only carrier pGluc-basic-2-CMV was transfected at equal total plasmid content as compared to the $\mathrm{U} 3$ mini-gene transfection.

U3 snoRNA promoter-reporter assay. The human U3 snoRNA gene promoter sequence was synthesized (Genecust, Boynes, France) from the 500 nucleotide sequence upstream of the U3 snoRNA transcription start site of SNORD3A gene (Ensembl) and cloned into the pNL1.2[NlucP] vector (Promega). pNL1.2[NlucP]_ Hs_U3 was transfected into non-OA HACs or SW1353 cells (seeded at 15,000 cells/ $\mathrm{cm}^{2}$ ) with Fugene6 and reseeded $2 \mathrm{~h}$ after transfection. U3 snoRNA promoter-reporter cells were exposed to OA synovial fluid (OA-SF; $20 \%(\mathrm{v} / \mathrm{v})$, pool of six donors), corresponding control (20\% (v/v) of $0.9 \% \mathrm{NaCl}), \mathrm{BMP} 7$ (R\&D Systems, Minneapolis, Minnesota, USA), IL1 $\beta$ (Life Technologies, Waltham, Massachusetts, USA) or TNFa (Sigma-Aldrich, Dorset, UK). For bioluminescence analysis cells were lysed with lysis buffer (Promega) and promoter-activity was measured with the Nano-Glo Luciferase Assay System (Promega) on a Tristar LB 942 (Berthold, Bad Wildbad, Germany). DNA-content was measured using a SYBR-GREEN (Invitrogen) assay. Relative differences were determined as compared to control conditions following correction for background and normalization by DNA content.

Gene expression. Cells were rinsed with $0.9 \% \mathrm{NaCl}$ and cell lysis, $\mathrm{RNA}$ isolation and subsequent cDNA synthesis were performed using the Cells-to-Ct kit (ThermoFisher Scientific, Waltham, Massachusetts, USA). Alternatively, cells were disrupted with TRIzol reagent (Life Technologies), followed by RNA isolation and quantification and cDNA synthesis as described previously ${ }^{20}$. The real-time quantitative polymerase chain reaction was performed as described previously ${ }^{5}$ and using validated primer sequences described in Supplementary Table 3. Ct-values were analyzed with the standard curve method and RNA (including non-coding- and messenger-RNA) expression was normalized to cyclophilin expression or the relative DNA-content between treatments. DNA-content for normalization was measured in parallel wells receiving the identical treatment. DNA-content was measured using a DAPI and HOECHST staining further explained in "SUNsET-assay". Differential gene expression was determined as a fold change compared to control conditions.

In-solution tryptic digestion and mass spectrometry proteomics. Cell lysates following U3 snoRNA knockdown (or control) in SW1353 cells were used ${ }^{21}$. Cells were harvested from triplicate wells of a six well plate with parallel wells used for confirmation of U3 snoRNA knockdown. In-solution tryptic digestion on $10 \mu \mathrm{g}$ protein was undertaken as previously described ${ }^{22}$. For the secretomes in-solution tryptic digestion was carried out on $10 \mu \mathrm{l}$ of StrataClean resins (Agilent Genomics, 400,714) on $100 \mu \mathrm{g}$ of protein for each sample as described previously ${ }^{23}$. Liquid chromatography-tandem mass spectrometry (LC-MS/MS) analysis was performed on trypsin digests using an Ultimate 3,000 Nanosystem (Dionex, ThermoFisher Scientific) online to a Q-Exactive Quadrupole-Orbitrap instrument (Thermo Scientific) ${ }^{24}$. Proteins were identified using an in-house Mascot server (Matrix Science, London, UK). Search parameters used were as follows: enzyme; trypsin, peptide mass tolerances $10 \mathrm{ppm}$, fragment mass tolerance of $0.01 \mathrm{Da}, 1+, 2+$, and $3+$ ions, with carbamidomethyl cysteine as a fixed modification and methionine oxidation as a variable modification, searching against the UniHuman Reviewed database, with a false discovery rate (FDR) of $1 \%$, a minimum of two unique peptides per protein.

Label-free quantification of mass spectrometry proteomics data. Progenesis QI software (version 4, Waters, Manchester, UK) was used to identify fold changes in protein abundance between U3 snoRNA knockdown and scrambled ASO control condition ${ }^{25}$. Only unique peptides were used for quantification, and with p-values $<0.05$, were considered to be differentially expressed (DE). Proteomic data has been deposited in the PRIDE ProteomeXchange and can be accessed using the identifier PXD017253 ${ }^{26}$.

Ingenuity pathway analysis. Functional analysis of differentially expressed proteins following U3 knockdown in SW1353 cells was undertaken to evaluate the differences in protein abundance. Ingenuity pathway analysis has been performed as previously described ${ }^{27}$.

Protein translation capacity. For a puromycilation assay ${ }^{28,29}$, cultures were incubated for 15 min with $5.4 \mu \mathrm{M}$ puromycin (Sigma-Aldrich, Dorset, UK), immediately followed by washing steps with PBS and fixation for 20 min with $10 \%$ formalin (VWR). Wells were washed with PBS-T and treated for 10 min with $0.1 \%$ Triton X-100. Wells were blocked for $1.5 \mathrm{~h}$ with $1 \%(\mathrm{~m} / \mathrm{v})$ skimmed milk powder (ELK, Campina, Zaltbommel, the Netherlands) in PBS-T, followed by overnight incubation at $4{ }^{\circ} \mathrm{C}$ with the anti-puromycin antibody $12 \mathrm{D} 10$ 
(Sigma-Aldrich, Dorset, UK). After washing with PBS-T, wells were incubated for $1 \mathrm{~h}$ at room temperature with goat anti-mouse Alexa488 (ThermoFisher Scientific). Following a final wash step, the fluorescence signal intensity was determined using a Tristar LB942 (Berthold, Bad Wildbad, Germany) equipped with excitation filter F485 and emission filter F353. Fluorescence data were normalized to DNA-content ${ }^{30}$. For that, the same wells were subsequently washed with HEPES-Buffered Saline (HBS), followed by $1 \mathrm{~h}$ incubation with $5 \mu \mathrm{g} / \mathrm{mL}$ DAPI (Invitrogen) plus $5 \mu \mathrm{g} / \mathrm{mL}$ HOECHST 33342 (Invitrogen) in HBS. After washing steps with HBS, fluorescence signal intensity was determined using a Tristar LB942 (Berthold), using the excitation filter F355 and emission filter F460.

RNA electrophoresis and northern blotting. Two micrograms total RNA was separated on a 6\% PAAgel (6\% PAA (Acrylamide/Bis-acrylamide 30:1); 1×TBE (Tris-borate-EDTA; Merck, Darmstadt, Germany); $8 \mathrm{M}$ urea), electro-transferred to a Hybond-N membrane (GE Healthcare; Chicago, Illinois, USA) and immobilized by UV-crosslinking (CL-1000, UVP). Membrane was pre-hybridized for $1 \mathrm{~h}$ at $62^{\circ} \mathrm{C}$ under agitation in hybridization buffer $(2 \times$ SSC, $5 \times$ Denhardts solution, $200 \mu \mathrm{g} / \mathrm{ml}$ fish sperm DNA, $0.1 \%$ SDS $)$. Membrane was subsequently hybridized overnight with the U3 snoRNA probe $(5 \mathrm{nM}$; Supplementary Table 4$)$ at $62{ }^{\circ} \mathrm{C}$ under agitation. Non-hybridized probe was rinsed from the membrane using a series of SSC-SDS wash buffers. Membrane was then incubated in blocking buffer [ $1 \times$ PBS, $0.5 \%$ SDS, $0.1 \%$ I-Block (ThermoFisher Scientific)] for $30 \mathrm{~min}$, followed by $30 \mathrm{~min}$ incubation in blocking buffer containing $0.2 \mu \mathrm{g} / \mathrm{ml}$ Streptavidin-AP (Life Technologies). After washing with PBS the membrane was equilibrated with detection buffer (100 mM Tris- $\mathrm{HCl} \mathrm{pH} \mathrm{9.5;}$ $100 \mathrm{mM} \mathrm{NaCl} ; 1 \mathrm{mM} \mathrm{MgCl}$ ) and detection was carried out using CDP-Star (Sigma-Aldrich, Dorset, UK) and visualized/quantified using a Bio-Rad Chemidoc MP imaging system. U6 snRNA was detected as a reference RNA on the same membrane by reprobing and repeating the procedure in-full while using the U6 snRNA probe. Full length images of northern blots are shown in Supplementary Fig. 4. The U3 snoRNA signal was normalized by the signal for U6 snRNA and relative differences between conditions were calculated.

Statistical analyses. Statistical significance has been calculated using GraphPad Prism software version 5.0 (La Jolla, California, USA) using paired or unpaired (depending on experiment) Student's t-test . Depending on the experiment this was done 1-tailed or 2-tailed. Details per experiment are indicated in the corresponding figure legends. Conditions were compared to control and statistical significance was set at $\mathrm{P}<0.05$. To test for normal distribution of the input data, D'Agostino-Pearson omnibus normality tests were performed. All quantitative data sets presented here passed the normality tests. Bars in graphs represent mean \pm standard deviation (SD).

\section{Results}

U3 snoRNA expression is decreased in cartilage and chondrocytes as function of $O A$. In order to find clues whether ribosome biogenesis is disturbed in osteoarthritic cartilage at the snoRNA level, a previously performed miRNA microarray experiment on young non-OA cartilage and old OA cartilage ${ }^{19}$, on which probe sets for snoRNAs were also present, was reanalyzed (Supplementary Fig. 1). A number of snoRNAs was found to be differentially expressed between non-OA and OA cartilage and amongst these U3 snoRNA expression was significantly lower in OA cartilage (Fig. 1A). Since U3 is one of the most abundant snoRNAs playing a key role in ribosome biogenesis, we investigated its involvement in chondrocyte homeostasis in more detail. To verify whether isolated OA chondrocytes have decreased U3 snoRNA levels, we measured U3 snoRNA expression in chondrocytes isolated from young non-OA and from old OA cartilage. Expression of U3 snoRNA in chondrocytes from OA cartilage was reduced (Fig. 1B) compared to non-OA chondrocytes. Reduced U3 snoRNA expression in OA chondrocytes was accompanied with a distinctive cellular phenotype associated with (pre-)hypertrophic OA chondrocytes ${ }^{5,31}$, with significantly reduced mRNA expression of chondrogenic genes COL2A1; ACAN; SOX9 and NKX3-2 and increased expression of chondrocyte hypertrophy-associated genes COL10A1; RUNX2; ALPL; MMP13; ADAMTS5; COX2 and IL6. Since there was an age difference between the non-OA cartilage/chondrocytes and the OA cartilage/chondrocytes used to determine differences in U3 snoRNA expression (Fig. 1A/B), we next measured OA-dependent alterations in chondrocyte U3 snoRNA levels in an experimental mouse model for traumatic OA (destabilization of medial meniscus; $\mathrm{DMM}^{32}$ ). Eight weeks post-DMM surgery knees were processed for U3 snoRNA in situ hybridization (ISH). A general reduction of U3 snoRNA ISH signal was observed in articular chondrocytes within the weight-bearing area of the joint in DMM-operated compared to sham-operated knees (Fig. 1C; red arrows). This was also observed in the menisci (Fig. 1C; blue arrowheads). U3 snoRNA levels were unaltered in chondrocytes in non-weight-bearing areas of the knee joints in sham-operated versus DMM-operated-mice (Fig. 1C; black arrows). Next, we asked whether OA-like conditions are capable of reducing U3 snoRNA expression levels in chondrocytes. To this end, non-OA human articular chondrocytes (HACs) from different donors were exposed to $20 \%$ (v/v) OA synovial fluid. U3 snoRNA expression was significantly diminished in HACs exposed to OA synovial fluid (Fig. 1D). Exposing non-OA HACs to non-OA synovial fluid caused a significant increase in the expression of U3 snoRNA (Fig. 1E). A U3 snoRNA gene promoter-reporter assay in SW1353 cells (Fig. 1F) or in HACs (Fig. 1G) revealed that OA synovial fluid reduced U3 snoRNA promoter transcriptional activity in SW1353 cells and two out of three HAC donors. In addition we observed that U3 snoRNA promotor transcriptional activity is reduced in HACs by exposure to the two katabolic cytokines IL1 $\beta$ (Fig. 1H) or TNFa (Fig. 1I). Collectively, these data suggest that OA conditions are able to impact U3 snoRNA levels in chondrocytes.

U3 snoRNA levels influence the articular chondrocyte's transcriptomic phenotype. It is unknown whether the level of U3 snoRNA expression influences the chondrocyte phenotype on the tran- 

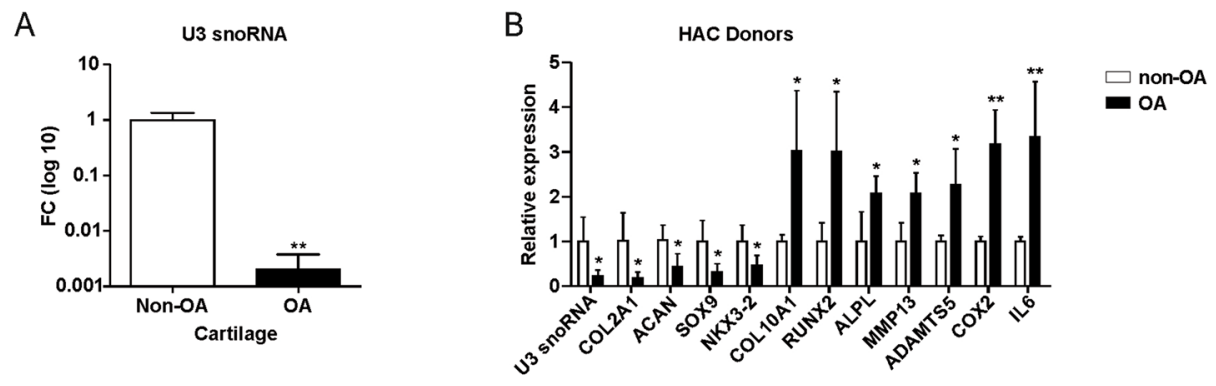

C

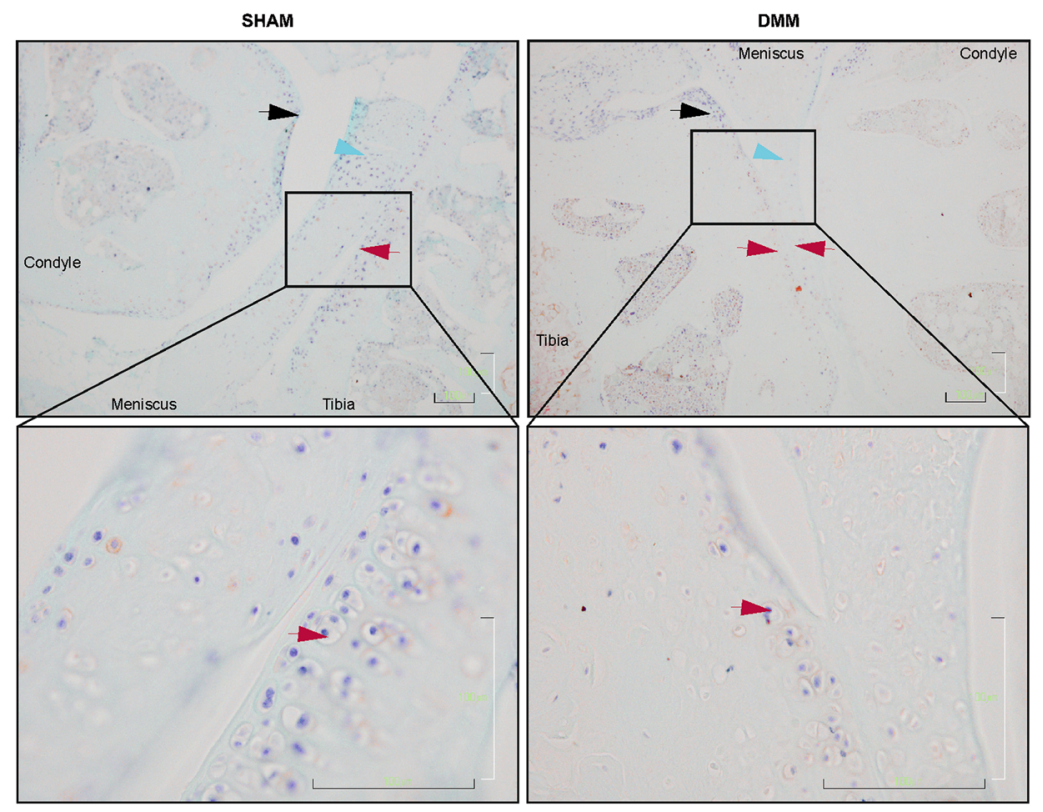

D

E

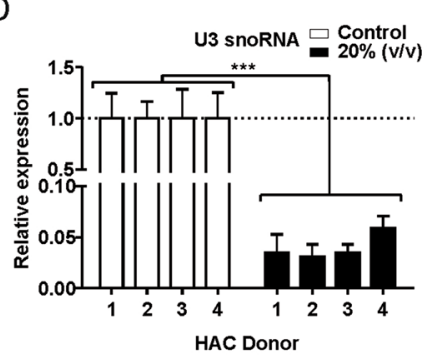

G

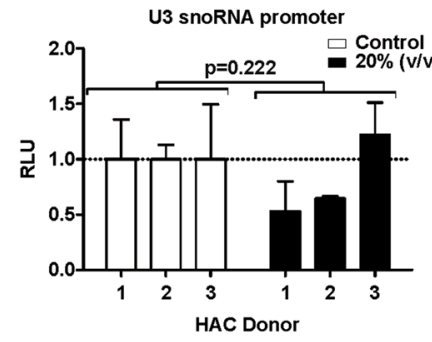

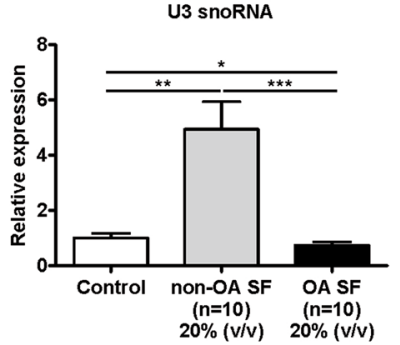

$\mathrm{H}$

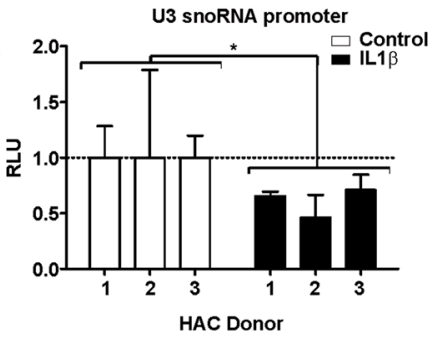

F

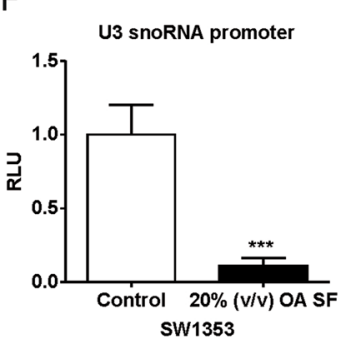

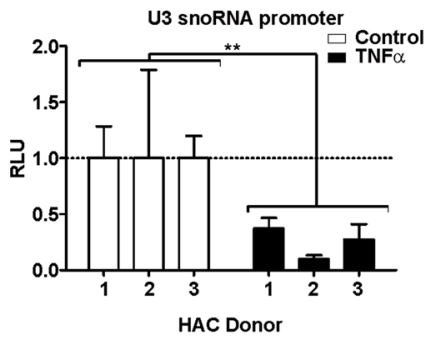


4 Figure 1. Impaired expression of U3 snoRNA in osteoarthritic cartilage and chondrocytes. (A) Total RNA from non-OA or OA cartilage ( $\mathrm{n}=6$ donors per group) was extracted and hybridized onto Affymetrix miRNA 4.0 arrays. Using a probe set for Homo sapiens, differential expression of ncRNAs was determined (Supplementary Fig. 1). Expression of U3 snoRNA in microarray data is depicted as Log 10 fold-change. (B) Expression levels of U3 snoRNA; mRNA expression of chondrogenic genes (COL2A1; ACAN; SOX9; NKX3-2), hypertrophic genes (RUNX2; COL10A1, ALPL), matrix-degrading enzyme genes (MMP13, ADAMTS5) and inflammatory mediator genes (COX2; IL6) were determined in chondrocytes derived from OA donors relative to non-OA controls $(\mathrm{n}=4$ donors per group) using RT-qPCR analysis. Data were normalized to cyclophilin expression. (C) DMM $(n=3)$ or sham surgery $(n=3)$ was performed in C57BL6/J male mice. Eight weeks post-surgery mice were sacrificed and knees prepared for U3 snoRNA ISH (representative images are depicted). Bottom images show the field-of-view in the boxes from the upper images at higher magnification. Red arrow heads: load-bearing articular cartilage surfaces; black arrow heads: non-load-bearing articular cartilage surfaces; blue arrow heads: meniscus. (D) Non-OA chondrocytes ( $\mathrm{n}=4$ donors) were cultured for $24 \mathrm{~h}$ in the presence or absence of OA synovial fluid (OA SF) (20\% (v/v); pool of 6 donors)). Expression of U3 snoRNA was determined relative to non-treated controls by RT-qPCR analysis. Results were normalized to total DNA content in parallel wells. (E) Non-OA chondrocytes ( $\mathrm{n}=4$ donors) were cultured for $24 \mathrm{~h}$ in the presence or absence of synovial fluid from non-OA (non-OA SF) or OA (OA SF) donors [20\% (v/v); pool of ten donors)]. Expression of U3 snoRNA was determined relative to control condition by RT-qPCR analysis. Data were normalized to cyclophilin expression. (F-I) SW1353 cells or non-OA HACs ( $\mathrm{n}=3$ donors) were transfected with a pNL1.2[NlucP]_Hs_U3_promoter plasmid. Subsequently, cells were exposed to OA synovial fluid ( $20 \%(\mathrm{v} / \mathrm{v})$, a pool of six donors), IL1 $\beta$ (10 ng/ $\mathrm{ml})$ or TNFa $(20 \mathrm{ng} / \mathrm{ml})$ for $20 \mathrm{~h}$ and Nanoluc luciferase levels were measured. Data were calculated relative to control conditions (RLU). Statistical significance was determined using Student's t-tests; (A/B/E-I) 2-tailed unpaired, (D) 2-tailed paired. Bars show the mean $( \pm \mathrm{SD}) .{ }^{*} \mathrm{P}<0.05,{ }^{* *} \mathrm{P}<0.01,{ }^{* *} \mathrm{P}<0.001$ versus control conditions.

scriptomic level. Therefore we altered U3 snoRNA expression levels in non-OA primary chondrocytes using a U3 snoRNA-specific antisense oligonucleotide (ASO). Following ASO transfections in five individual nonOA chondrocyte cultures, reduced U3 snoRNA expression was confirmed (Fig. 2A). As a result, expression of COL2A1, SOX9, NKX3-2, RUNX2, MMP13 and IL6 was significantly reduced in all five chondrocyte isolates (Fig. 2B-G), but with variable levels of change. Reciprocally, we ectopically increased expression of U3 snoRNA moderately by transfecting primary chondrocytes with a U3 mini-gene (Fig. 3). The activity of the U3 mini-gene was confirmed by northern blot (Fig. 3A) and significantly elevated U3 snoRNA expression was confirmed in primary chondrocytes (Fig. 3B). The elevated U3 snoRNA expression levels resulted in upregulated levels of mRNAs coding for COL2A1 and NKX3-2 (Fig. 3C/E), while expression of SOX9 and IL6 transcripts was reduced (Fig. 3D/H). Expression of RUNX2 and MMP13 was also altered following ectopic expression of U3 snoRNA, albeit with strong inter-donor variability (Fig. 3F/G). Taken together, we demonstrated that ectopically-induced alterations in the expression levels of U3 snoRNA in articular chondrocytes change the chondrocyte's transcriptomic phenotype.

Chondrocyte rRNA levels are reduced in OA conditions and are regulated by U3 snoRNA. U3 snoRNA is rate-limiting in the generation of mature rRNAs ${ }^{12,33}$ and $18 \mathrm{~S}$ rRNA in particular. To determine whether rRNA levels are altered in primary chondrocytes in OA conditions, we measured 18S, 5.8S and $28 \mathrm{~S}$ rRNA expression. In concert with reduced U3 snoRNA levels in old OA chondrocytes (Fig. 1A/B), we observed reduced expression levels of $18 \mathrm{~S}$ and $5.8 \mathrm{~S}$ rRNAs in old OA chondrocytes, while $28 \mathrm{~S}$ rRNA levels remained unaltered (Fig. 4A). Following the exposure of HACs to OA synovial fluid we observed a reduction of $18 \mathrm{~S}$ and $5.8 \mathrm{~S}$ transcript levels (Fig. 4B), while $28 \mathrm{~S}$ rRNA expression was not significantly altered. OA synovial fluid also reduced expression of $18 \mathrm{~S}$ and 5.8S rRNAs in HACs (Fig. 4B), while an opposite response of 18S and 5.8S rRNA levels was detected when HACs were exposed to non-OA synovial fluid (Fig. 4B). In both conditions $28 \mathrm{~S}$ rRNA expression was not significantly changed. Alteration of U3 snoRNA expression in primary chondrocytes (Figs. 2 and 3) led to changes in chondrocyte rRNA levels. Reduction of U3 led to reduced 18S and 28S rRNA levels, with a sharp decrease of 5.8S rRNA levels in four out of five chondrocyte donors (Fig. 4C). Ectopic U3 expression led to increased $18 \mathrm{~S}$ and $28 \mathrm{~S}$ rRNA levels (Fig. 4D), while not changing 5.8S rRNA levels in three out of four chondrocyte donors. Overall, data demonstrate that $18 \mathrm{~S}$ and $5.8 \mathrm{~S}$ rRNA levels in chondrocytes are susceptible to OA conditions and rRNA transcript levels respond to U3 snoRNA expression.

U3 snoRNA levels influence the activity of the chondrocyte protein translation apparatus. Our data demonstrate alterations in chondrocyte rRNA levels in OA conditions and following knockdown or ectopic expression of U3 snoRNA. Since rRNAs are the catalytically active subunits of the translating ribosome, we next investigated the consequences for overall protein translational capacity. Protein translational capacity was determined in chondrocytes from young non-OA and old OA cartilage and a significantly reduced translational capacity was observed in osteoarthritic chondrocytes (Fig. 5A). Translational capacity of chondrocyte cultures with diminished U3 snoRNA expression was significantly reduced (Fig. 5B), while ectopic induction of U3 snoRNA expression resulted in a mild, but significantly increased translational capacity (Fig. 5C). These data show that U3 snoRNA is able to change the chondrocyte's translational capacity. To determine the full impact of U3 snoRNA knockdown and the concomitant reduction in rRNA levels on the chondrocyte translational apparatus, we subsequently conducted whole proteome analysis on SW1353 cells in which U3 snoRNA expression was reduced. A reduction of $\mathrm{U} 3$ snoRNA and concomitant reduction in $18 \mathrm{~S}$ and $5.8 \mathrm{~S}$ rRNA levels 
A

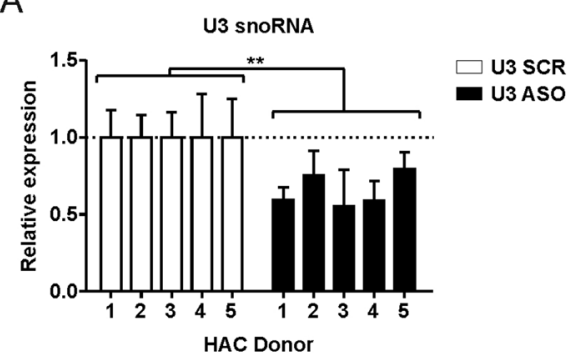

B

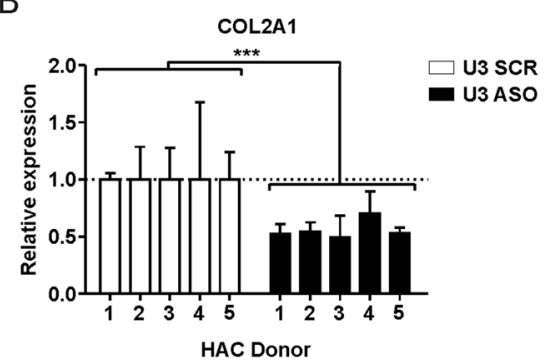

$E$

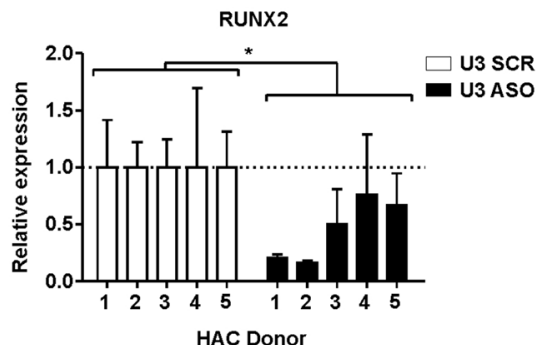

C

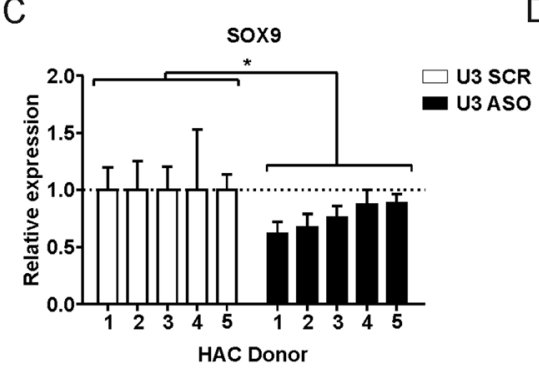

$\mathrm{F}$

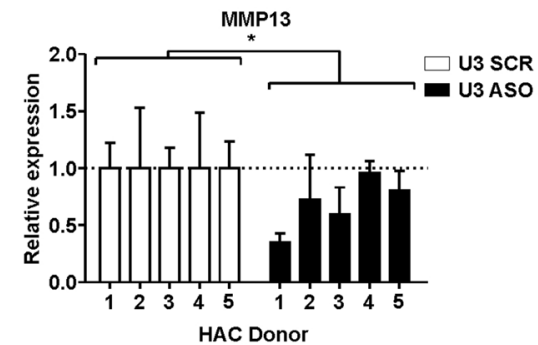

D

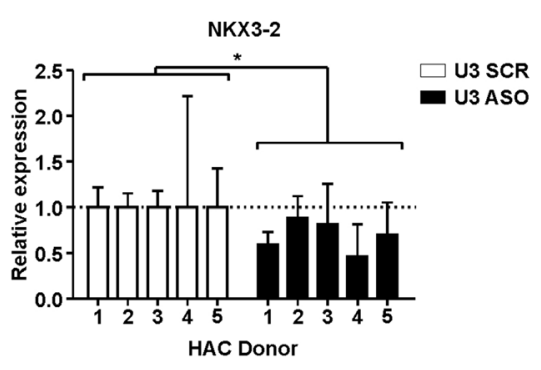

G

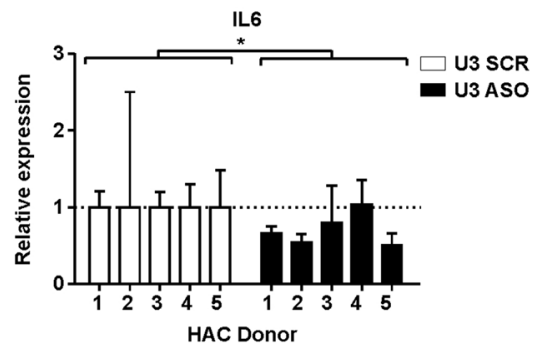

Figure 2. U3 snoRNA knock-down impacts the articular chondrocyte's phenotype. Non-OA chondrocytes ( $\mathrm{n}=5$ donors) were transfected with a U3 snoRNA ASO (U3 ASO) or scrambled (SCR) ASO and cultured for 24 h. (A) Expression of U3 snoRNA; (B) COL2A1; (C) SOX9; (D) NKX3-2; (E) RUNX2; (F) MMP13 and (G) IL6 mRNA levels were determined by RT-qPCR analysis. Data from U3 ASO samples were calculated relative to controls transfected with the SCR ASO. Results were normalized to relative total DNA content in parallel wells. Statistical significance was determined using 2-tailed paired Student's t-tests. Bars show the mean $( \pm S D)$. ${ }^{*} \mathrm{P}<0.05,{ }^{*} \mathrm{P}<0.01,{ }^{* * *} \mathrm{P}<0.001$ versus control conditions.

were confirmed (Supplementary Fig. 2). Ingenuity Pathway Analysis (IPA) analysis of the differential proteomes revealed that IPA networks "synthesis of protein", "translation of protein", "metabolism of protein" and "initiation of translation of mRNA" were top deregulated networks (Fig. 5D) and confirm a global impact of the reduction of U3 snoRNA expression on protein translational processes. Also networks involving transcription and expression of RNA were prominently represented in the IPA analysis. The differential proteome that is observed following U3 snoRNA reduction concerns a particular global upregulation of differentially expressed proteins (Supplementary Fig. 3), rather than a downregulation and contains a great number of protein species involved in the process of protein translation and ribosomal stress responses. An additional proteomic analysis of the secretome of these U3 snoRNA knockdown cells was conducted. Relevant to inflammatory responses in OA cartilage, we could demonstrate that secreted proteins involved in antagonizing inflammatory processes were present in the secretomes of U3 knockdown cells in lower abundance (Fig. 5E). Together these data show alterations in chondrocyte protein translational activity following alterations in U3 snoRNA expression levels, with U3 snoRNA impacting protein translation molecular routes.

U3 snoRNA expression is increased by BMP7. We previously demonstrated that bone morphogenetic protein 7 (BMP7) is capable of ameliorating the OA chondrocyte phenotype ${ }^{5}$. Considering this anabolic action of BMP7 we evaluated whether U3 snoRNA and its subsequent rRNA targets can be induced in chondrocytes by BMP7. A U3 snoRNA promoter-reporter experiment in SW1353 cells or in non-OA HACs demonstrated that U3 snoRNA promoter transcriptional activity was induced following stimulation with BMP7 (Fig. 6A/B). In both chondrocyte cell models BMP7 induced U3 gene expression and increased expression of $18 \mathrm{~S}$ and $5.8 \mathrm{~S}$ rRNAs (Fig. 6C/D). The expression of $28 \mathrm{~S}$ rRNA was not significantly altered. SW1353 cells stimulated with 
A

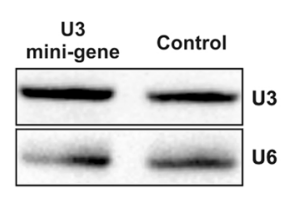

C

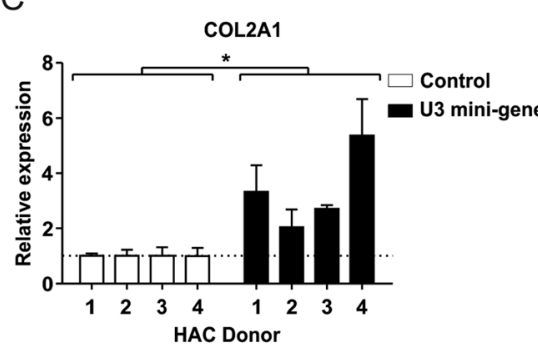

$\mathrm{F}$

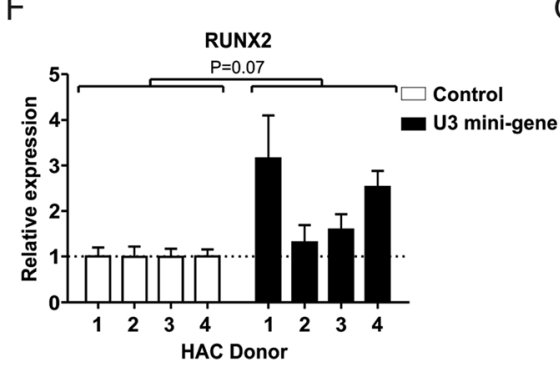

U3 snoRNA

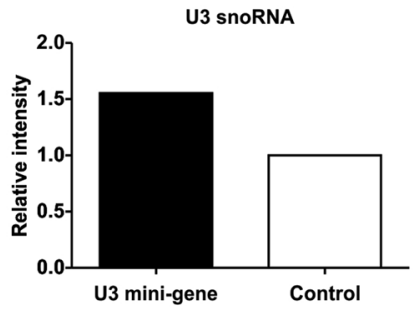

D
B

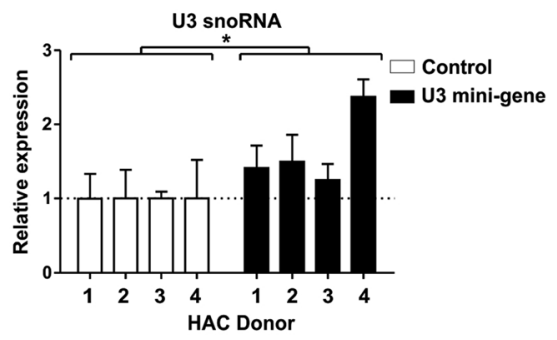

E

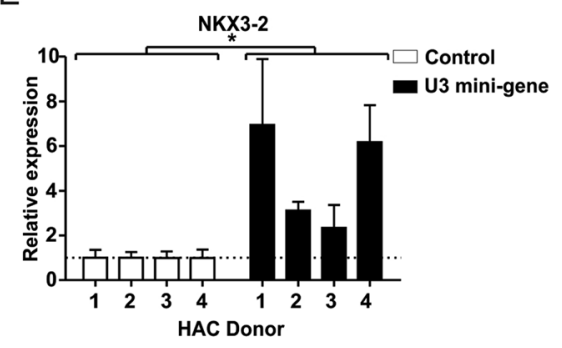

$\mathrm{H}$

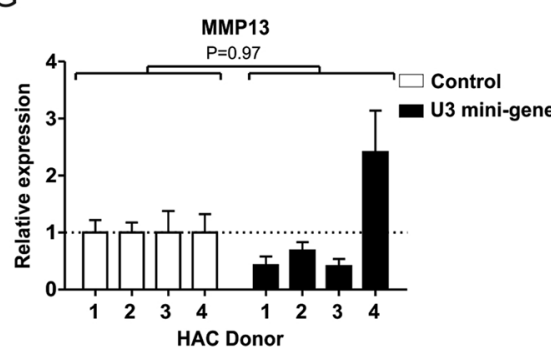

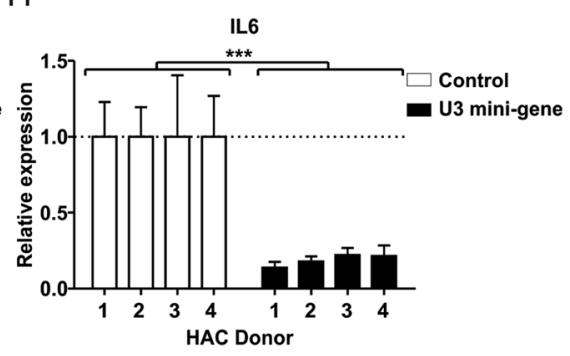

Figure 3. Ectopic expression of U3 snoRNA impacts the chondrocyte's phenotype. (A) Activity of the U3 minigene in primary chondrocytes was confirmed by northern blot $(n=1)$ left panel. U3 snoRNA expression levels were calculated relative to control and normalized for U6 snRNA, right panel. Non-OA chondrocytes $(n=4$ donors) were transfected with the U3 mini-gene $\left(10 \mathrm{ng}\right.$ plasmid/ $\left./ \mathrm{cm}^{2}\right)$ and cultured for $24 \mathrm{~h}$. (B) Expression levels of U3 snoRNA; (C) COL2A; (D) SOX9; (E) NKX3-2; (F) RUNX2; (G) MMP13 and (H) IL6 gene expression levels were determined relative to control. Results were normalized to total DNA content in parallel wells. Statistical significance was determined using Student's t-tests; (B) 1-tailed paired, (C-H) 2-tailed paired. Bars show the mean $( \pm \mathrm{SD}) .{ }^{*} \mathrm{P}<0.05,{ }^{* *} \mathrm{P}<0.01,{ }^{* * *} \mathrm{P}<0.001$ versus control conditions.

BMP7 had an increased translational capacity (Fig. 6E), supporting the notion that the BMP7-mediated induction of U3 snoRNA and rRNA expression increases the chondrocyte's translational capacity.

\section{Discussion}

U3 snoRNA is an abundant snoRNA with a pivotal function in the generation of mature $18 \mathrm{~S}$ rRNA in eukaryotes $^{14}$. It's snoRNP biochemistry ${ }^{34}$ and mechanism-of-action ${ }^{35}$ have been extensively studied in the past decades. However, it was only recently described to be univocal involved in $18 \mathrm{~S}$ rRNA generation in human cells $^{12}$. In contrast with its decade-long thorough molecular characterization, insight into its involvement in human disease is limited to a role in cell proliferation and tumorigenesis ${ }^{12,36}$, while it remained unknown whether U3 snoRNA levels can be regulated via extracellular cues.

We demonstrate that in different osteoarthritic conditions the expression level of U3 snoRNA in articular chondrocytes is diminished. Reduced expression of U3 snoRNA was previously reported in serum of ageing mice $^{37}$, and as a result of nucleus pulposus ageing in mice ${ }^{38}$. As a general study limitation in this field, we could not investigate the potential influence of age difference on U3 snoRNA expression between non-OA and OA cartilage/chondrocytes. To circumvent potential U3 expression interpretation issues, we reinforced our analyses by performing additional U3 snoRNA expression analyses in a murine model for OA and used synovial fluid from $\mathrm{OA}$ and non-OA individuals (no statistically significant age difference) on chondrocytes. Both as models in which age was not a potential confounder. Data from these models confirmed the OA-dependent reduction of chondrocyte U3 snoRNA expression. We could also conclude that reduced OA chondrocyte U3 snoRNA expression is, at least in part, mediated via diminished U3 gene transcription activity. This is the first data demonstrating that the extracellular environment is capable of controlling cellular U3 snoRNA levels, thereby tuning the cell's capacity to generate mature rRNA species. U3 snoRNA is transcribed from a dedicated transcriptional unit under the control of an RNA polymerase II-driven (RNAPII) promoter. Little is known about the control 

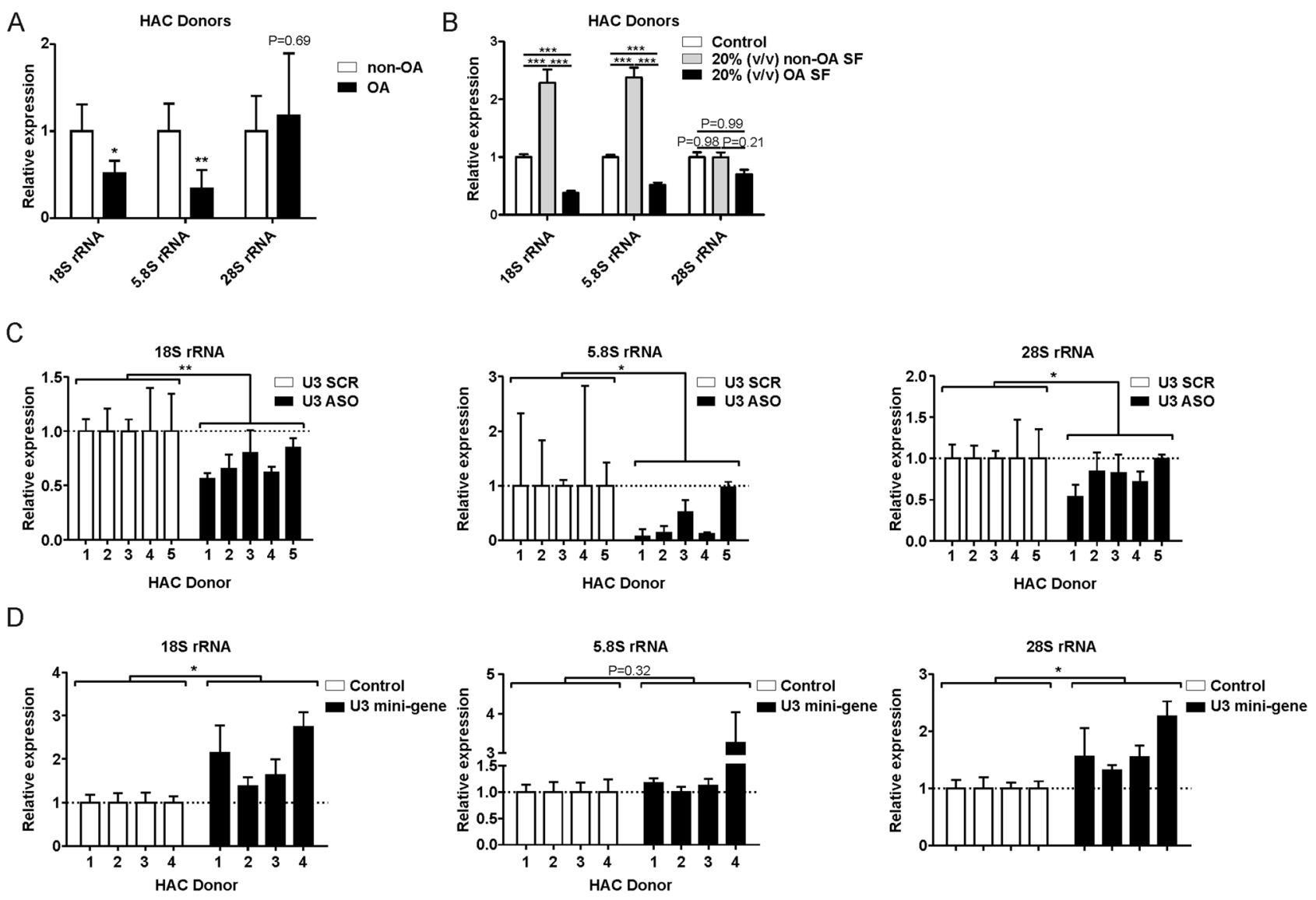

Figure 4. Chondrocyte ribosomal RNA expression is impacted in OA conditions and following alteration of U3 snoRNA levels. (A) rRNA expression levels were determined in OA and non-OA primary chondrocytes ( $\mathrm{n}=4$ donors per group). (B) Non-OA chondrocytes ( $\mathrm{n}=4$ donors) were cultured for $24 \mathrm{~h}$ in the presence or absence of synovial fluid from non-OA or OA donors [20\% (v/v); pool of ten donors)]. Expression of rRNAs was determined relative to control condition by RT-qPCR analysis. Data were normalized to cyclophilin expression. (C) Non-OA chondrocytes ( $\mathrm{n}=5$ donors) were transfected with a U3 snoRNA ASO or SCR ASO and cultured for $24 \mathrm{~h}$ and rRNA levels were measured using RT-qPCR. (D) Non-OA chondrocytes ( $\mathrm{n}=4$ donors) were transfected with a U3 mini-gene (10 ng plasmid $\left./ \mathrm{cm}^{2}\right)$ and cultured for $24 \mathrm{~h}$ and $\mathrm{rRNA}$ levels were determined by RT-qPCR. Data were normalized to cyclophilin $(\mathbf{A} / \mathbf{B})$ or to relative total DNA content in parallel wells (C/D). Statistical significance was determined using Student's t-tests; (A/B) 2-tailed unpaired, (C/D) 2-tailed paired. Bars show the mean $( \pm \mathrm{SD}) .{ }^{\star} \mathrm{P}<0.05,{ }^{* *} \mathrm{P}<0.01,{ }^{* *} \mathrm{P}<0.001$ versus control conditions.

of snoRNA transcription from dedicated transcriptional units, but our group previously reported that transcriptional activity of another dedicated snoRNA gene (RMRP) can also be influenced via major chondrocyte signaling pathways during chondrogenesis ${ }^{39}$. OA synovial fluid is a complex body fluid containing a plethora of catabolic morphogens and cytokines/chemokines ${ }^{40,41}$. Therefore we expect that the signaling molecule composition of OA synovial fluid acts on major chondrocyte signaling pathways, leading to the observed decreased U3 snoRNA expression. Indeed our data demonstrated that the katabolic cytokines IL1 $\beta$ and TNFa attenuated U3 promoter activity. Reciprocally, we demonstrate that non-OA synovial fluid and BMP7 are capable of inducing U3 snoRNA transcription and subsequent U3 snoRNA levels in chondrocytes.

A network of snoRNAs is involved in key ribosome biogenesis processes ${ }^{16}$, and U3 snoRNA is a factor in the generation of $18 \mathrm{~S} \mathrm{rRNA}^{14}$. Indeed our data show that manipulation of chondrocyte U3 snoRNA levels impacts $18 \mathrm{~S}$ rRNA levels, but also alters $5.8 \mathrm{~S}$ and $28 \mathrm{~S}$ rRNA levels in many instances. Taking into consideration the $47 \mathrm{~S}$ pre-rRNA multi-cistronic origin of $18 \mathrm{~S}$ rRNA, we speculate that aberrations in $18 \mathrm{~S}$ rRNA levels may impact 5.8S and $28 \mathrm{~S}$ rRNA levels possibly due to a $40 \mathrm{~S}$ over $60 \mathrm{~S}$ ribosomal subunit imbalance ${ }^{42}$. Also other snoRNAs involved in $47 \mathrm{~S}$ pre-rRNA processing could be impacted by OA conditions. Our unpublished data suggest deregulated chondrocyte U13 and RMRP snoRNA levels in OA conditions. This may provide additional explanations for the observed abberations in the levels of other rRNAs. In contrast with our current findings, other recent work demonstrated increased protein translation activity in OA cartilage ${ }^{43-45}$. The OA cartilage used in our study was derived from end-stage (K\&L grade 3-4) knee OA patients, while the OA grade of the human OA-lesioned cartilage presented by others ${ }^{45}$ appears moderate. Taking into account that the reported increased protein translation activity in a rat model for OA seems to fade with progression into late OA development ${ }^{45}$, we speculate that the contradictory observations may be explained by OA severity of the analyzed cartilage. This is further supported by the idea that an inflammatory component is much more dominant in early OA than in end-stage $\mathrm{OA}^{46}$, and it 
A

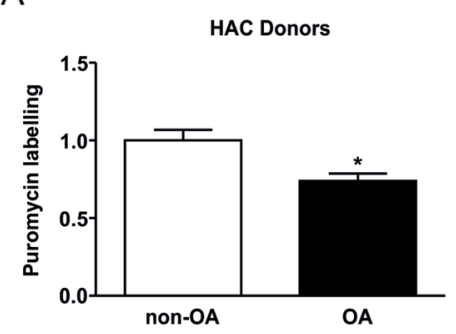

B

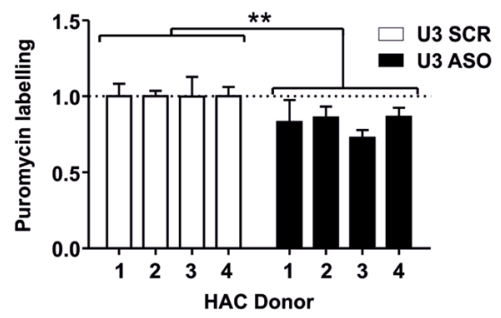

C

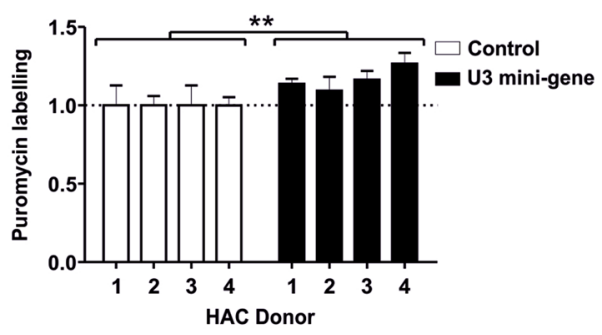

$\mathrm{D}$

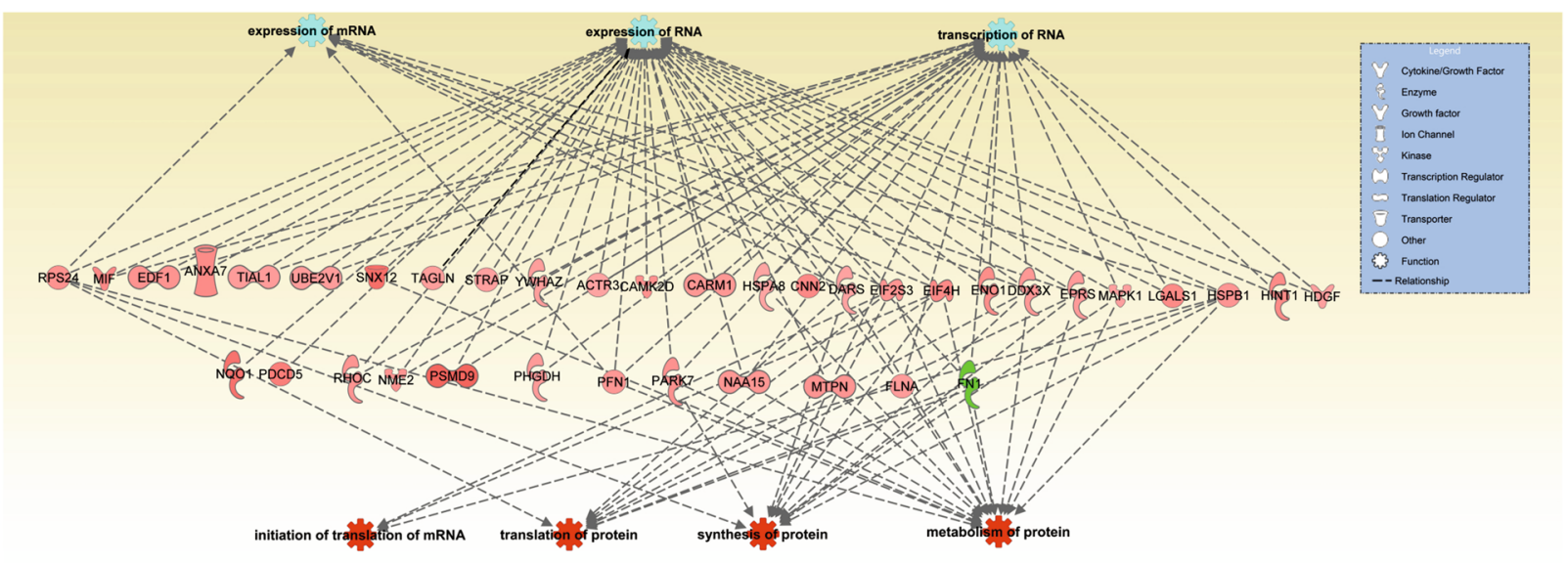

$E$

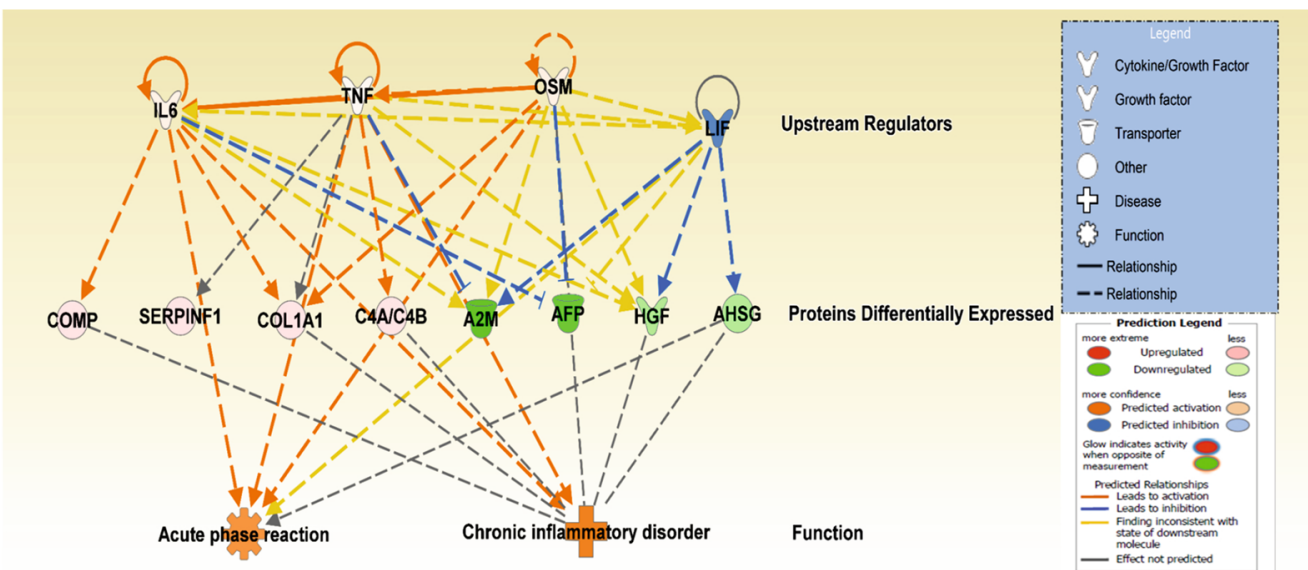

Figure 5. U3 snoRNA expression levels influence the chondrocyte protein translation capacity and its proteome. Protein translational capacity was determined using a puromycilation assay in a number of conditions: (A) OA or non-OA primary chondrocytes ( $n=4$ donors per group), (B) in U3 snoRNA knockdown conditions in non-OA HACs ( $\mathrm{n}=4$ donors) transfected with a U3 snoRNA ASO or SCR ASO, (C) and in non-OA primary chondrocytes $\left(\mathrm{n}=4\right.$ donors) transfected with a $\mathrm{U} 3 \mathrm{mini}$-gene $\left(10 \mathrm{ng} \mathrm{plasmid} / \mathrm{cm}^{2}\right)$. Puromycilation data $(\mathbf{A}-\mathbf{C})$ were calculated relative to corresponding controls and were normalized to DNA content. Statistical significance was determined using Student's t-tests; (A) 2-tailed unpaired, (B/C) 2-tailed paired. Bars show the mean $( \pm \mathrm{SD}) .{ }^{\star} \mathrm{P}<0.05,{ }^{*} \mathrm{P}<0.01,{ }^{* *} \mathrm{P}<0.001$ versus control conditions. (D) SW1353 cells were transfected with a U3 ASO or SCR control and cultured for $24 \mathrm{~h}$ (knockdown was confirmed in Supplementary Fig. 2), after which LC-MS/MS and label-free quantification was conducted. Ingenuity pathway analysis was performed on the differentially expressed proteins between conditions. Green nodes; decreased expression following knockdown, red nodes; increased expression following knockdown. Intensity of colour is related to a higher fold-change. Key to the main features in the networks is shown. (E) Upstream analysis in Ingenuity Pathway Analysis on the differentially expressed secreted proteins between conditions [(D) U3 snoRNA knockdown in SW1353]. This analyses linkage to differentially expressed proteins through coordinated expression, identify potential upstream regulators that has been observed experimentally to affect gene expression. Green nodes; decreased expression following U3 snoRNA knockdown, red nodes; increased expression following knockdown. Intensity of colour is related to a higher fold-change. Key to the main features in the networks is shown. 
A

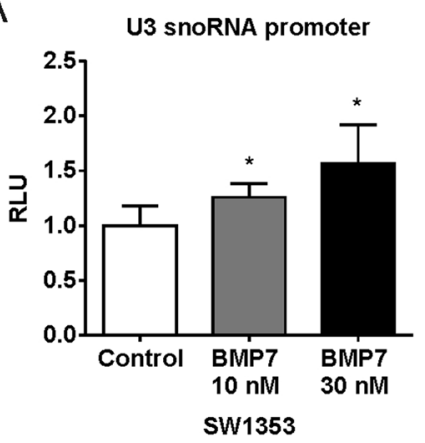

C

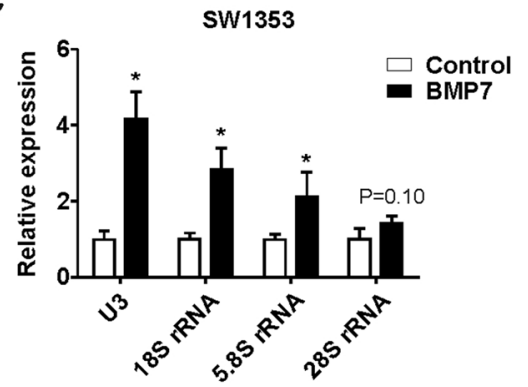

$\mathrm{B}$

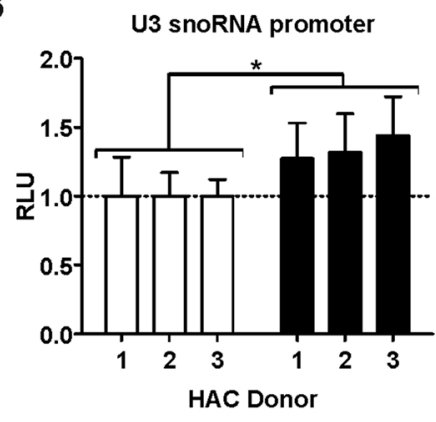

D

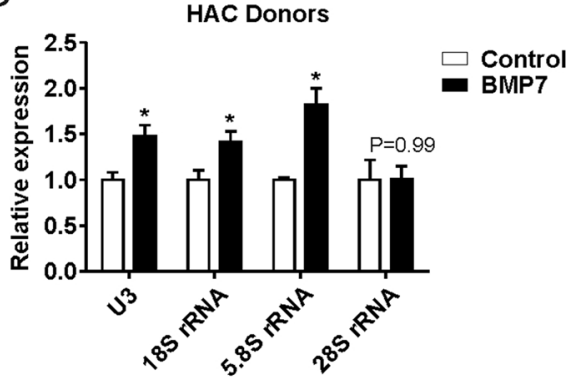

$E$

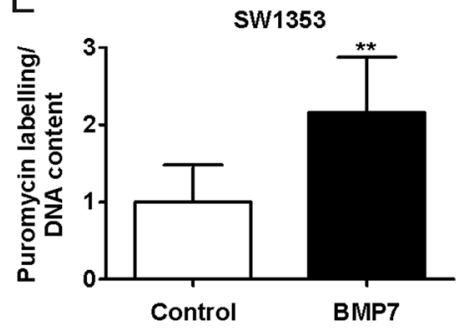

Figure 6. U3 snoRNA and rRNA expression levels are regulated by BMP7. (A) SW1353 cells or (B) non-OA primary chondrocytes $(n=3)$ were transfected with a pNL1.2[NlucP]_Hs_U3_promoter plasmid. Subsequently, SW1353 cells (A) were exposed to 10 or $30 \mathrm{nM}$ BMP7 and HACs (B) to $10 \mathrm{nM} \mathrm{BMP7}$ for $20 \mathrm{~h}$ and Nanoluc luciferase levels were measured. Data were normalized and calculated relative to control conditions (RLU). (C) SW1353 cells and (D) non-OA primary chondrocytes $(n=4)$, were exposed to $1 \mathrm{nM} \mathrm{BMP7}$ for 24 h. Expression of U3 snoRNA; 18S, 5.8S and 28S rRNA was determined relative to control conditions by RT-qPCR analysis. Results were normalized to cyclophilin expression. (E) SW1353 cells were exposed to $1 \mathrm{nM} \mathrm{BMP7} \mathrm{for} 24 \mathrm{~h}$. Protein translation capacity was determined using a puromycilation assay and data were normalized for DNA content. Statistical significance was determined using Student's t-tests; (A/B) 1-tailed unpaired, (C/E) 2-tailed unpaired, (D) 2-tailed paired. Bars show the mean $( \pm \mathrm{SD}) .{ }^{\star} \mathrm{P}<0.05,{ }^{\star *} \mathrm{P}<0.01,{ }^{* *} \mathrm{P}<0.001$ versus control conditions.

was shown that the increase in translation activity could be recapitulated by IL1 $\beta^{45}$. This suggests that an increase in chondrocyte translation activity in OA may be predominantly related to early OA, while impaired protein translation may be a hallmark of end-stage OA.

While increased chondrocyte protein synthesis capacity in early OA may be related to mTOR signaling and imbalanced autophagy ${ }^{47}$, it is unknown what the specific consequence is of a diminished capacity of global protein synthesis in relation to development or progression of osteoarthritis. We propose that U3 snoRNA-related reduction of chondrocyte protein translation affects the chondrocyte's ability to maintain the molecular build-up of the cartilage proteinaceous ECM. Alterations in protein translation capacity may render articular cartilage vulnerable to any biomechanical, catabolic, traumatic or age-related changes in the joint that are associated with OA development and progression ${ }^{2}$. In addition, our gene expression data, which are a representation of the overall chondrocyte differentiation status ${ }^{3,4}$, show that alterations in chondrocyte U3 snoRNA levels affects its differentiation status. Data from the secretome proteomics analysis further show U3-dependent alterations in the levels of secreted proteins involved in balancing inflammatory pathways (A2M; AFP; HGF; AHSG). Together this shows that besides an influence on protein translation, alterations in chondrocyte U3 snoRNA levels additionally impact the chondrocyte's homeostatic integrity.

Our whole proteome analysis uncovered that reduction of U3 snoRNA levels induces ribosome stress, which is indicated by aberrations in molecular pathways controlling key aspects of rRNA and ribosomal protein gene transcription (PGAM1; EDF1; MAPK1; AHCY; CARM1; FLNA), rRNA processing (FSCN1; RPS8; RAN; RPS24), ribosomal subunit transport (RAN), translation initiation (PHGHD; AKT1S1; EIF4H; ARPC5; TIAL1; EIF2S3; PABPC1; HSPB1; TPT1; FN1), chain elongation (DARS; EPRS; DDX3X) and protein folding/ chaperoning (AHSA1; HSPA8; HSP90; HSPB1) (Supplementary Fig. 3). Ribosome stress following reduction of U3 snoRNA expression was previously reported and is accompanied by stabilization of $\mathrm{p} 53^{12}$. Indeed we also observed increased expression of p53 after U3 snoRNA knockdown in SW1353 cells (data not shown). It was previously reported that p53 expression is also increased in OA chondrocytes ${ }^{48}$. Stabilisation of p53 feeds back to ribosome biogenesis and down regulates translational activity ${ }^{49,50}$. Besides the U3 snoRNA-dependent availability of rRNAs for ribosome biogenesis, this may provide a link between our observed OA-related impairment of U3 snoRNA expression levels in chondrocytes and their translational activity. In addition, IPA analyses of the U3 knock-down cellular proteome demonstrated that a great number of the differentially expressed proteins are targets of Myc (data not shown). We therefore speculate that stabilization of p53 may impact chondrocyte gene 
transcription processes via Myc, providing a potential explanation for the impact and discrepancies found in chondrocyte gene expression following alteration of U3 levels (Figs. 2, 3).

To comprehend the implications of the larger snoRNA network ${ }^{16}$ on the function of the articular chondrocyte, other members of the non-canonical and canonical classes of snoRNAs should also be investigated. However, our study for the first time demonstrates the involvement of a snoRNA in articular chondrocyte biology. The OA-related impaired expression of U3 snoRNA has detectable consequences for articular chondrocyte protein translation capacity, rendering U3 snoRNA expression a potential therapeutic target in OA treatment. Indeed our data show that U3 snoRNA expression can be induced by the growth factor BMP7, providing a potential manner to target U3 snoRNA in OA chondrocytes.

Received: 19 April 2020; Accepted: 23 July 2020

Published online: 10 August 2020

\section{References}

1. Loeser, R. F., Collins, J. A. \& Diekman, B. O. Ageing and the pathogenesis of osteoarthritis. Nat. Rev. Rheumatol. 12, 412-420. https ://doi.org/10.1038/nrrheum.2016.65 (2016).

2. Loeser, R. F., Goldring, S. R., Scanzello, C. R. \& Goldring, M. B. Osteoarthritis: A disease of the joint as an organ. Arthritis Rheum. 64, 1697-1707. https://doi.org/10.1002/art.34453 (2012).

3. Ripmeester, E. G. J., Timur, U. T., Caron, M. M. J. \& Welting, T. J. M. Recent insights into the contribution of the changing hypertrophic chondrocyte phenotype in the development and progression of osteoarthritis. Front. Bioeng. Biotechnol. 6, 18. https://doi. org/10.3389/fbioe.2018.00018 (2018).

4. van der Kraan, P. M. \& van den Berg, W. B. Chondrocyte hypertrophy and osteoarthritis: Role in initiation and progression of cartilage degeneration?. Osteoarthr. Cartil. 20, 223-232. https://doi.org/10.1016/j.joca.2011.12.003 (2012).

5. Caron, M. M. et al. BAPX-1/NKX-3.2 acts as a chondrocyte hypertrophy molecular switch in osteoarthritis. Arthritis Rheumatol. (Hoboken, N.J.) 67, 2944-2956. https://doi.org/10.1002/art.39293 (2015).

6. Chen, J. \& Long, F. mTOR signaling in skeletal development and disease. Bone Res. 6, 1-1. https://doi.org/10.1038/s41413-0170004-5 (2018).

7. Rellmann, Y. \& Dreier, R. Different forms of ER stress in chondrocytes result in short stature disorders and degenerative cartilage diseases: New insights by cartilage-specific ERp57 knockout mice. Oxidat. Med. Cell. Longevity 2018, 8421394. https://doi. org $/ 10.1155 / 2018 / 8421394$ (2018).

8. Uehara, Y. et al. Endoplasmic reticulum stress-induced apoptosis contributes to articular cartilage degeneration via C/EBP homologous protein. Osteoarthr. Cartil. 22, 1007-1017. https://doi.org/10.1016/j.joca.2014.04.025 (2014).

9. Khatter, H., Myasnikov, A. G., Natchiar, S. K. \& Klaholz, B. P. Structure of the human 80 S ribosome. Nature 520, 640-645. https ://doi.org/10.1038/nature14427 (2015).

10. Henras, A. K., Plisson-Chastang, C., O’Donohue, M. F., Chakraborty, A. \& Gleizes, P. E. An overview of pre-ribosomal RNA processing in eukaryotes. Wiley Interdiscip. Rev. RNA 6, 225-242. https://doi.org/10.1002/wrna.1269 (2015).

11. Mullineux, S. T. \& Lafontaine, D. L. Mapping the cleavage sites on mammalian pre-rRNAs: Where do we stand?. Biochimie 94, 1521-1532. https://doi.org/10.1016/j.biochi.2012.02.001 (2012).

12. Langhendries, J.-L., Nicolas, E., Doumont, G., Goldman, S. \& Lafontaine, D. L. J. The human box C/D snoRNAs U3 and U8 are required for pre-rRNA processing and tumorigenesis. Oncotarget 7, 59519-59534. https://doi.org/10.18632/oncotarget.11148 (2016).

13. Dupuis-Sandoval, F., Poirier, M. \& Scott, M. S. The emerging landscape of small nucleolar RNAs in cell biology. Wiley Interdiscip. Rev. RNA 6, 381-397. https://doi.org/10.1002/wrna.1284 (2015).

14. Beltrame, M. \& Tollervey, D. Base pairing between U3 and the pre-ribosomal RNA is required for $18 \mathrm{~S}$ rRNA synthesis. EMBO J. 14, 4350-4356 (1995).

15. Kass, S., Tyc, K., Steitz, J. A. \& Sollner-Webb, B. The U3 small nucleolar ribonucleoprotein functions in the first step of preribosomal RNA processing. Cell 60, 897-908. https://doi.org/10.1016/0092-8674(90)90338-F (1990).

16. Lafontaine, D. L. J. Noncoding RNAs in eukaryotic ribosome biogenesis and function. Nat. Struct. Molecular Biol. 22, 11. https:// doi.org/10.1038/nsmb.2939 (2015).

17. Kung, L. H. W., Rajpar, M. H., Preziosi, R., Briggs, M. D. \& Boot-Handford, R. P. Increased classical endoplasmic reticulum stress is sufficient to reduce chondrocyte proliferation rate in the growth plate and decrease bone growth. PLoS ONE 10, e0117016. https ://doi.org/10.1371/journal.pone.0117016 (2015).

18. Yamabe, S. et al. Intracellular accumulation of advanced glycation end products induces apoptosis via endoplasmic reticulum stress in chondrocytes. FEBS J. 280, 1617-1629. https://doi.org/10.1111/febs.12170 (2013).

19. Balaskas, P. et al. MicroRNA profiling in cartilage ageing. Int. J. Genom. 2017, 2713725. https://doi.org/10.1155/2017/2713725 (2017).

20. Caron, M. M. J. et al. Hypertrophic differentiation during chondrogenic differentiation of progenitor cells is stimulated by BMP-2 but suppressed by BMP-7. Osteoarthr. Cartil. 21, 604-613. https://doi.org/10.1016/j.joca.2013.01.009 (2013).

21. Peffers, M. J., Collins, J., Loughlin, J., Proctor, C. \& Clegg, P. D. A proteomic analysis of chondrogenic, osteogenic and tenogenic constructs from ageing mesenchymal stem cells. Stem Cell Res. Ther. 7, 133. https://doi.org/10.1186/s13287-016-0384-2 (2016).

22. Peffers, M. J., Beynon, R. J. \& Clegg, P. D. Absolute quantification of selected proteins in the human osteoarthritic secretome. Int. J. Mol. Sci. 14, 20658-20681. https://doi.org/10.3390/ijms141020658 (2013).

23. Kharaz, Y. A. et al. Comparison between chaotropic and detergent-based sample preparation workflow in tendon for mass spectrometry analysis. Proteomics https://doi.org/10.1002/pmic.201700018 (2017).

24. Anderson, J. R. et al. The synovial fluid proteome differentiates between septic and nonseptic articular pathologies. J. Proteom. 202, 103370. https://doi.org/10.1016/j.jprot.2019.04.020 (2019).

25. Peffers, M. J. et al. Proteomic analysis reveals age-related changes in tendon matrix composition, with age- and injury-specific matrix fragmentation. J. Biol. Chem. 289, 25867-25878. https://doi.org/10.1074/jbc.M114.566554 (2014).

26. Vizcaino, J. A. et al. 2016 update of the PRIDE database and its related tools. Nucleic Acids Res. 44, D447-456. https://doi. org/10.1093/nar/gkv1145 (2016).

27. Peffers, M., Liu, X. \& Clegg, P. Transcriptomic signatures in cartilage ageing. Arthritis Res. Therapy 15, R98. https://doi.org/10.1186/ $\operatorname{ar} 4278(2013)$.

28. Goodman, C. A. \& Hornberger, T. A. Measuring protein synthesis with SUnSET: A valid alternative to traditional techniques?. Exerc. Sport Sci. Rev. 41, 107-115. https://doi.org/10.1097/JES.0b013e3182798a95 (2013).

29. Henrich, C. J. A Microplate-based nonradioactive protein synthesis assay: Application to TRAIL sensitization by protein synthesis inhibitors. PLoS ONE 11, e0165192. https://doi.org/10.1371/journal.pone.0165192 (2016). 
30. McCaffrey, T. A., Agarwal, L. A. \& Weksler, B. B. A rapid fluorometric DNA assay for the measurement of cell density and proliferation in vitro. In Vitro Cell. Dev. Biol. J. Tissue Culture Assoc. 24, 247-252. https://doi.org/10.1007/bf02623555 (1988).

31. Ji, Q. et al. Single-cell RNA-seq analysis reveals the progression of human osteoarthritis. Ann. Rheum. Dis. 78, 100-110. https:// doi.org/10.1136/annrheumdis-2017-212863 (2019).

32. Culley, K. L. et al. Mouse models of osteoarthritis: Surgical model of posttraumatic osteoarthritis induced by destabilization of the medial meniscus. Methods Mol. Biol. (Clifton, N.J.) 1226, 143-173. https://doi.org/10.1007/978-1-4939-1619-1_12 (2015).

33. Panse, V. G. \& Johnson, A. W. Maturation of eukaryotic ribosomes: Acquisition of functionality. Trends Biochem. Sci. 35, 260-266. https://doi.org/10.1016/j.tibs.2010.01.001 (2010).

34. Dragon, F. et al. A large nucleolar U3 ribonucleoprotein required for $18 \mathrm{~S}$ ribosomal RNA biogenesis. Nature 417, 967-970. https ://doi.org/10.1038/nature00769 (2002).

35. Granneman, S. et al. Role of pre-rRNA base pairing and $80 \mathrm{~S}$ complex formation in subnucleolar localization of the U3 snoRNP. Mol. Cell. Biol. 24, 8600-8610. https://doi.org/10.1128/mcb.24.19.8600-8610.2004 (2004).

36. Ismael, H., Altmeyer, S. \& Stahl, H. Regulation of the U3-, U8-, and U13snoRNA expression by the DEAD box proteins Ddx5/ Ddx17 with consequences for cell proliferation and survival. Non-coding RNA. https://doi.org/10.3390/ncrna2040011 (2016).

37. Steinbusch, M. M. F. et al. Serum snoRNAs as biomarkers for joint ageing and post traumatic osteoarthritis. Sci. Rep. 7, 43558. https://doi.org/10.1038/srep43558 (2017).

38. Veras, M. A., McCann, M. R., Tenn, N. A. \& Seguin, C. A. Transcriptional profiling of the murine intervertebral disc and ageassociated changes in the nucleus pulposus. Connect Tissue Res. 61, 63-81. https://doi.org/10.1080/03008207.2019.1665034 (2020).

39. Steinbusch, M. M. F. et al. Expression of RMRP RNA is regulated in chondrocyte hypertrophy and determines chondrogenic differentiation. Sci. Rep. 7, 6440. https://doi.org/10.1038/s41598-017-06809-5 (2017).

40. Balakrishnan, L. et al. Proteomic analysis of human osteoarthritis synovial fluid. Clin. Proteom. 11, 6-6. https://doi. org/10.1186/1559-0275-11-6 (2014).

41. Anderson, J. R. et al. (1)H NMR metabolomics identifies underlying inflammatory pathology in osteoarthritis and rheumatoid arthritis synovial joints. J. Proteome Res. 17, 3780-3790. https://doi.org/10.1021/acs.jproteome.8b00455 (2018).

42. Gregory, B. et al. The small and large ribosomal subunits depend on each other for stability and accumulation. Life Sci. Alliance 2, e201800150. https://doi.org/10.26508/lsa.201800150 (2019).

43. Sandell, L. J. Anabolic factors in degenerative joint disease. Curr. Drug Targets 8, 359-365. https://doi.org/10.2174/1389450077 79940142 (2007).

44. Henrotin, Y. \& Reginster, J. Y. Anabolic events in osteoarthritis. Osteoarthr. Cartil. 7, 310-312. https://doi.org/10.1053/ joca.1998.0175 (1999).

45. Katsara, O., Attur, M., Ruoff, R., Abramson, S. B. \& Kolupaeva, V. Increased activity of the chondrocyte translational apparatus accompanies osteoarthritic changes in human and rodent knee cartilage. Arthritis Rheumatol. Hoboken NJ 69, 586-597. https:// doi.org/10.1002/art.39947 (2017).

46. Sokolove, J. \& Lepus, C. M. Role of inflammation in the pathogenesis of osteoarthritis: Latest findings and interpretations. Ther. Adv. Musculoskelet. Dis. 5, 77-94. https://doi.org/10.1177/1759720X12467868 (2013).

47. Zhang, Y. et al. Cartilage-specific deletion of mTOR upregulates autophagy and protects mice from osteoarthritis. Ann. Rheum. Dis. 74, 1432-1440. https://doi.org/10.1136/annrheumdis-2013-204599 (2015).

48. Hashimoto, S. et al. Role of $\mathrm{p} 53$ in human chondrocyte apoptosis in response to shear strain. Arthritis Rheum. 60, 2340-2349. https://doi.org/10.1002/art.24706 (2009).

49. Marcel, V., Van Long, F. N. \& Diaz, J. J. 40 years of research put p53 in translation. Cancers. https://doi.org/10.3390/cancers100 $50152(2018)$.

50. Golomb, L., Volarevic, S. \& Oren, M. p53 and ribosome biogenesis stress: The essentials. FEBS Lett. 588, 2571-2579. https://doi. org/10.1016/j.febslet.2014.04.014 (2014).

\section{Acknowledgements}

This work was financially supported by the Dutch Arthritis Association (Grants LLP14 and 17-2-401), a Wellcome Trust Clinical Intermediate Fellowship 107471/Z/15/Z, and by the Medical Research Council (MRC) and Versus Arthritis as part of the Medical Research Council Versus Arthritis Centre for Integrated Research into Musculoskeletal Ageing (CIMA) [MR/R502182/1]. The MRC Versus Arthritis Centre for Integrated Research into Musculoskeletal Ageing is a collaboration between the Universities of Liverpool, Sheffield and Newcastle. The funders had no role in study design, data collection and analysis, decision to publish, or preparation of the manuscript. The authors are grateful to Dr. P. Emans, Dr. P. Feczko and Dr. T. Boymans for their support in collecting the OA patient material.

\section{Author contributions}

Substantial contributions to research design: E.G.R., L.W.R, M.M.J.C., M.J.P., T.J.M.W. Substantial contributions to the acquisition of samples: E.G.R., G.G.H.A., D.A.M.S., A.C., P.B., P.D., B.A.C.H., A.C., A.S., Y.F., M.M.J.C., M.J.P. Substantial contributions to analysis: E.G.J.R., G.G.H.A., D.A.M.S., A.C., P.B., P.D., B.A.C.H., A.C., A.S., Y.F., M.M.J.C., M.P. Substantial contributions to the interpretation of data: E.G.J.R., G.G.H.A., D.A.M.S., A.C., P.B., P.D., B.A.C.H., A.C., A.S., Y.F., L.W.R., M.M.J.C., M.P., T.J.M.W. Drafting the paper: E.G.J.R., L.W.R., M.M.J.C., M.J.P., T.J.M.W. Revising paper critically: E.G.J.R., G.G.H.A., D.A.M.S., A.C., P.B., P.D., B.A.C.H., A.C., A.S., Y.F., L.W.R., M.M.J.C., M.J.P., T.J.M.W. Approval of the submitted and final versions: E.G.J.R., G.G.H.A., D.A.M.S., A.C., P.B., P.D., B.A.C.H., A.C., A.S., Y.F., L.W.R., M.M.J.C., M.J.P., T.J.M.W.

\section{Competing interests}

MMJ Caron and TJM Welting are inventor on patents WO2017178251 and WO2017178253 (licensed to Chondropeptix). LW van Rhijn and TJM Welting have shares in Chondropeptix and are CDO and CSO of Chondropeptix, respectively. The other authors have no competing interests.

\section{Additional information}

Supplementary information is available for this paper at https://doi.org/10.1038/s41598-020-70453-9.

Correspondence and requests for materials should be addressed to T.J.M.W.

Reprints and permissions information is available at www.nature.com/reprints. 
Publisher's note Springer Nature remains neutral with regard to jurisdictional claims in published maps and institutional affiliations.

(c) (i) Open Access This article is licensed under a Creative Commons Attribution 4.0 International License, which permits use, sharing, adaptation, distribution and reproduction in any medium or format, as long as you give appropriate credit to the original author(s) and the source, provide a link to the Creative Commons license, and indicate if changes were made. The images or other third party material in this article are included in the article's Creative Commons license, unless indicated otherwise in a credit line to the material. If material is not included in the article's Creative Commons license and your intended use is not permitted by statutory regulation or exceeds the permitted use, you will need to obtain permission directly from the copyright holder. To view a copy of this license, visit http://creativecommons.org/licenses/by/4.0/.

(c) The Author(s) 2020 